BLS 32, No 1 2006. DOI: http://dx.doi.org/10.3765/bls.v32i1.3443 (published by the Berkeley Linguistics Society and the Linguistic Society of America)

\title{
Methods for the Study of the Social Structure of Linguistic Variation
}

\author{
KATHRYN CAMPBELL-KIBLER \\ Stanford University
}

\section{Introduction}

Sociolinguists have long observed that linguistic variation correlates with a variety of social structures. In recent years, variation research has devoted increasing attention to social meaning, the idea that speakers use linguistic structures to build their identities and shape the situations and larger societal structures in which they participate. Although it is not usually presented in such terms, the concept of social meaning embodies a claim about cognition: linguistic variation and social practice not only correlate, but are linked, consciously or unconsciously, in speaker awareness.

To test this claim, I used the Matched Guise Technique (MGT), a tool developed by William Lambert and his colleagues in the 1960s (Lambert et al. 1960) to examine the English variable (ING) (e.g. working vs. workin'). In designing my study, I included a number of unusual and important features: using digitally manipulated speech, creating stimuli from spontaneous as opposed to read speech, and conducting both open-ended group interviews and a controlled experiment. The aim of these techniques is to uncover the social meaning of a single linguistic variable from the listener's point of view in a nuanced and rigorous manner. The next section will discuss the MGT literature, while Section 3 describes the key features I have incorporated into my study. I will then step through the details of the methods, describing in detail the procedures followed for generating the stimulus materials, manipulating the recordings, and collecting both the qualitative and quantitative data. Finally, I will briefly touch on the nature of the insights provided by these techniques.

\section{The Matched Guise Technique}

The study of language attitudes encompasses a range of approaches, including overt questioning, media analysis, and perceptual dialectology. The MGT was developed in the 1960s by William Lambert and his colleagues to covertly elicit individuals' attitudes towards members of different ethnolinguistic groups (Lambert et al. 1960). The technique involves having a single speaker produce 


\section{Kathryn Campbell-Kibler}

two (or more) utterances in different languages or varieties. Participants listen to the recordings and evaluate them on a range of qualities, such as how intelligent, educated, friendly, or trustworthy the speakers sound. Because listeners are not told that the alternate recordings have been produced by the same person, they evaluate each guise (language or accent performance) as an individual speaker. However, because the recordings have been produced by the same person, many of the paralinguistic cues are (hopefully) held constant, for example speech rate, pitch contours, and various aspects of voice quality. The content of the utterance is also usually held constant, so that any differences between the evaluations can be (in theory) assigned to different perceptions of the languages or varieties under study.

Research using the MGT has been carried out on a range of linguistic situations comparing multiple languages, such as French and English in Canada (Genesee and Holobow 1989), and language varieties, such as regional accents in England (Dixon et al. 2002). There has also been research into smaller units of difference, such as speech rate (Giles et al. 1992). Other work has employed the MGT to investigate evaluative reactions to specific sociolinguistic variables (Plichta and Preston 2005).

As the linguistic units of analysis become more detailed, we begin to stretch the notion of "attitude" to its limit. It may seem natural to talk of a listener's attitude towards French or a Southern accent. It is somewhat less so to speak of their attitude towards the length of $/ \mathrm{s} /$ in an utterance. If we are to successfully marry the fields of language attitudes and variation, a better understanding of the construct attitude is needed. Sociolinguists not only must understand the psychosocial significance of a listener's overall "attitude" towards a speaker, but also merge this understanding into our existing models of social meaning. This study documents a connection between the use of a given variant of (ING) in a specific situation and a change in the rating of a listener on a list of labeled scales. In social psychology terms, we are establishing information about the relative attitudes of the listener(s) to the two linguistic styles presented. This opens the question of how to translate that information into knowledge about the specific variables themselves and what the theoretical relationship is between the behavior changes registered by the measure and the concepts of "social meaning" or "indexing". This bridge is not yet built, but its construction represents the central theoretical work of the research program of which this project is a part.

\section{Methodological Choices}

The current study draws on a range of methodological techniques including the use of digitally manipulated stimuli, the use of speech from interviews rather than reading passages, the inclusion of multiple samples from each speaker, and the use of both open-ended interviews and a survey. Before going over the methods of my study in detail, I will touch on each of these tools to mention its previous uses and discuss its importance.

As advances in digital technology make it easier and less expensive to create 


\section{Methods for the Study of Linguistic Variation}

natural-sounding manipulated tokens, sociolinguists have become increasingly interested in creating MGT studies which explore specific variables. Thirty years ago, it was possible to use technology to change the rate, pitch, and pitch variation of a sample of speech (Brown et al. 1973). Today, we can use available software to alter the formants of a vowel (Graff et al. 1986, Fridland et al. 2004, Plichta and Preston 2005). While the current technology cannot yet directly alter a nasal, it does allow a "cut and paste" approach to produce a natural-sounding token, as in this project as well as Labov et al. (2005).

The benefit of digital manipulation is the precise control it gives. Without it, to construct matched stimuli one must have speakers perform each version, consciously shifting the variables as they speak. This can be straightforward when investigating attitudes towards whole languages or varieties, given access to perfectly bilingual or bidialectal speakers. When exploring more "molecular" variables (Scherer 1979), it becomes difficult to ensure that only the variable in question is affected. We understand very little about how different variables interact in the perception process, so it is unclear how far we can trust our judgments regarding whether other aspects of the performance have changed. We can still gather interesting and relevant information regarding the larger perceptual consequences of, say, fast or slow speech. What we cannot do is be sure what the precise linguistic triggers of this perceptual change are, something which is possible with more precise alteration.

Another advantage of digital manipulation is that by eliminating the need for conscious control on the part of the speaker it allows a wider range of speakers and speaking situations. It is possible to use naïve speakers, monolingual speakers and even, as here, samples from spontaneous speech. Much of the literature on the MGT has used read speech, although there have been exceptions (e.g. Wölck 1973, Apple et al. 1979, Graff et al. 1986). A significant body of work documents the differences between read and spontaneous speech with respect to prosody in particular (Hirose and Kawanami 2002). Further, listeners easily read these cues to recognize read speech (Levin et al. 1994). A few studies have shown that listener evaluations differ based on the reading/speaking distinction (Giles et al. 1980, Smith and Bailey 1980). This evidence makes it problematic to assume the results for read speech will reflect percepts in real, spontaneous interactions.

One of the potential drawbacks to using spontaneous speech is that it is difficult to control the content of the recordings. Unsurprisingly, the content of what speakers say impacts the judgments others make about them. Despite the control over the contrasting guises from the manipulation, the different speakers are presented saying different things. The effects of context are not eliminated, of course, in using read speech. Content and factors like word choice and sentence structure influence the interpretation of the speaker and may influence the role of other variables, including the ones of interest in a given study (see Giles et al. 1990). I included four recordings from each speaker, in order to begin to explore the role of content as well as provide some variation within the data from each speaker. Nonetheless, the content of the recordings varied widely across speakers, 


\section{Kathryn Campbell-Kibler}

and this variation had an impact on the results.

The final key methodological choice is the combination of qualitative data from group interviews with quantitative data from a survey-based experiment. Using multiple types of data helps to guard against the drawbacks characteristic of each while capitalizing on their strengths. Qualitative data is rich in nuance and provides insight into the ideologies and conscious thought processes involved in the evaluative process. The drawbacks are that these conscious processes may play a relatively minor role in the evaluative system, and the very richness of the data makes it difficult to be sure of the generalizability of the patterns found. Incorporating experimental data addresses both of these issues by collecting a larger and more easily quantified set of responses from listeners unaware of the linguistic features under study. The primary drawback of this data type, namely the difficulty of interpreting the reasoning behind the results, is countered by the interview data (Giles et al. 1990). Finally, by conducting interviews first, I was able to use them as a pilot to ensure a useful survey instrument (Williams et al. 1976).

The next section will be devoted to describing the methods of the study in detail, beginning with the creation and manipulation of the recordings, then describing the methods for data collection and finally the statistical analyses.

\section{The Study}

The existing literature and an early pilot for the current study suggested that there may be regional differences in the use and interpretation of (ING). Accordingly, I sought participants from two distinct areas in the U.S., one in the South (North Carolina) and one outside the South (California). Both speakers and listeners were university students. (1) gives the names (pseudonyms) of the speakers, divided by region and sex.

Speakers, by region and sex

\begin{tabular}{l|c|c} 
& Women & Men \\
\hline North Carolina & $\begin{array}{c}\text { Bonnie } \\
\text { Tricia }\end{array}$ & $\begin{array}{c}\text { Robert } \\
\text { Ivan }\end{array}$ \\
\hline California & $\begin{array}{c}\text { Elizabeth } \\
\text { Valerie }\end{array}$ & $\begin{array}{c}\text { Sam } \\
\text { Jason }\end{array}$
\end{tabular}

The speakers were all attending school in the state specified and had been raised in state, with the exception of the California speaker Elizabeth, who was originally from Seattle. All eight speakers were white.

\section{1. $\quad$ Creating the Stimuli}

To make the recordings, I met with each speaker twice, first for a sociolinguistic interview and again to generate (ING) tokens for splicing. The interview focused on schoolwork or other work experiences and their recreational activities and/or families. After each interview, I transcribed the resulting tape, then met again 


\section{Methods for the Study of Linguistic Variation}

with the speaker, explaining in more detail the point of the study, including the variable to be manipulated. I asked them to produce alternate tokens for each instance of (ING) from the original interview, both -in and -ing tokens in the same phrase used in the original.

Once the raw material was available, the next step was to select short clips to use in the study. I chose four passages from each speaker, two about school or work and two on recreational or family topics. Each excerpt was a single uninterrupted turn, with only the speaker's voice audible on the tape. In a handful of cases, I used adjacent turns of speech and clipped out an intervening prompt. The clips ranged from 10 to 20 seconds in length and contained two to six tokens of (ING) each. I used the software package Praat to digitally manipulate the selections, splicing in both -in and -ing variants, to minimize confounding differences introduced by the manipulation. (2) shows an image of the window used to select exactly the phonetic material to be cut and pasted.

Selecting the appropriate material: thing out of something

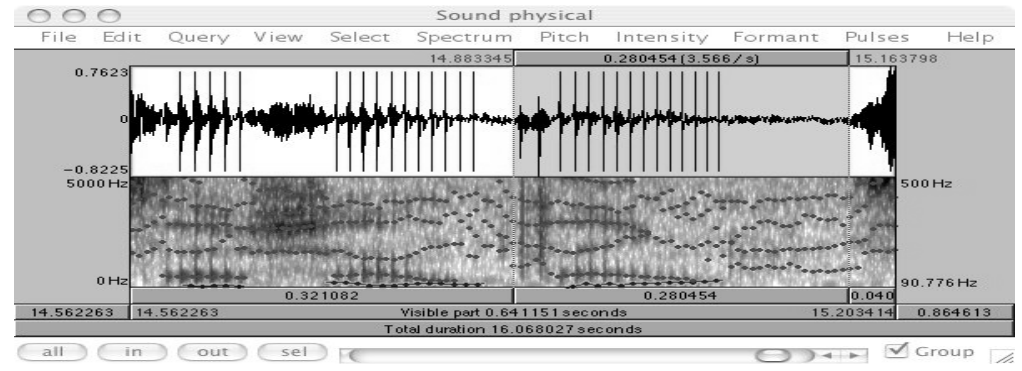

In selecting the points for splicing, I looked for either a pause, a stop closure, or a point within a stable sonorant. If it was necessary to make the change point in the middle of a sonorant, I used Praat's formant tracking feature to find points in each alternate and the original which were similarly located in time and matching roughly in their formant values. Once the boundaries of the three tokens were selected, I adjusted the tokens to be spliced in with respect to pitch, intensity, and length. I used Praat's facilities for examining and altering these qualities to match the alternates to the original as much as possible. I excerpted the pitch track from the original and used it to resynthesize the alternate tokens. I also altered the length of the alternates, usually to match the length of the original. In some cases, it was necessary to adjust the lengths of the vowel and nasal separately to match across tokens.

In other cases, the length or pitch of the two alternate tokens diverged so remarkably from the original that altering them both to match it resulted in a bizarre or impossible utterance. When this happened I placed priority on matching the manipulated alternates to each other and on obtaining a natural sound. The result often was that the newly manipulated recordings featured (ING) in a more promi- 
nent and easily heard setting than the original token from the interview, meaning the process changed the social content of the recording in a very real way.

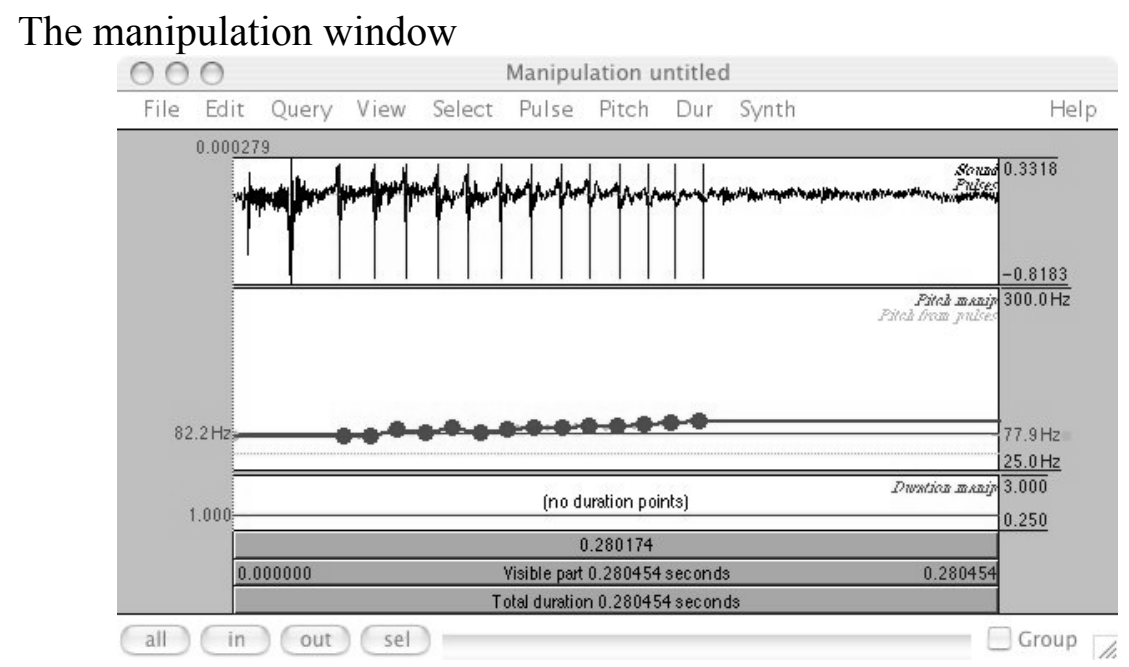

The issue of how similar the two members of the minimal pair needed to be was a tricky one. (ING) has been well studied as a sociolinguistic variable, but little work has been done concerning the specific phonetic attributes of the variants and how they differ, if at all. Under normal circumstances there may be regular differences between the two variants with respect to, for example, length. If there are such differences, it is not immediately clear what would constitute a minimal pair. On one hand, using the same lengths and pitches would create the most strictly similar tokens. On the other, if the normative lengths for the two variants differ, using identical lengths shifts the comparison to one between a typical token of one variant and an atypical token of another.

Once all of the recordings had been created, I ran a short pilot to confirm that the tokens were identifiable as the variants intended, using excerpts from the manipulated recordings, including a couple of words on each side of each token. Listeners were asked to identify whether each token was -in or -ing and to indicate whether the recording sounded strange. Each token was heard by at least five listeners, and tokens with more than two misclassifications or reports of strange sound were remanipulated and repiloted.

\section{Gathering Data}

Once the manipulated recordings were finished and tested, the next step was to collect open-ended responses. In conducting the group interviews I had two goals: to determine the general reactions to the speakers in each recording and the words used spontaneously to describe them, and to gather native speaker intuitions regarding (ING) and its effect on these particular utterances. In order to keep the 


\section{Methods for the Study of Linguistic Variation}

task a reasonable length, I divided the recordings by gender and content. One group heard only recordings of men discussing work-related topics, another heard men discussing recreational topics, and so on. The groups ranged in number from one-on-one interviews up to groups with six participants. The bulk of the interviews were with two or three participants.

I began by explaining the purpose of the study, that I was trying to learn about how small differences in the way we speak influence how we are perceived. For the first portion of the interview, I went through each of the four speakers, asking general questions: What can you tell me about Jason? Does he sound competent or good at what he does? Is he someone you would be likely to be friends with? Who do you think he's talking to? What is the context of the conversation? Where do you think he is from?

After going through the four speakers once, we listened to a second recording from each speaker in which they said different things on the same kind of topic, either work or recreation. The second recording for each speaker contained a different variant of (ING) than the first, but this was not brought to the attention of the participants. I asked participants to discuss if and how the second recording changed their impression of the speaker and whether the context seemed to be the same as the first recording.

Transitioning to the second half of the interview, I explained the goal of my study in more detail, introducing the variable (ING). I played each of eight recordings once more, this time with their opposing (ING) guise, explicitly identifying which guise we were hearing at each step. For each pair, I asked how the change in (ING) influenced their perception of the speaker, if at all. The whole interview took approximately one hour. In all, 20 groups consisting of 55 participants were analyzed, one group having been eliminated due to problems with the recording and another due to a preponderance of non-native speakers.

After completing and transcribing the interviews, I moved on to the experimental phase of the study. The interviews and the survey collected very different kinds of information. The interviews elicited explicit ideologies involving (ING) and reactions to the recordings overall. The goal of the survey was to investigate covert reactions to (ING), testing which meanings are actually influenced in online interpretation of speakers. The first step in developing the experimental design for the survey was analyzing the interview data for the descriptions to use in the survey. In selecting terms used in the interviews, I used two primary criteria. I sought the most central characterizations of the speakers, on the basis of which characterizations were most frequently mentioned, mentioned early on in the interviews, and frequently presupposed. I also selected terms which were frequently referenced in the discussions of (ING). Although I drew primarily on the interview data, I also combined these with concepts drawn from the production literature on (ING) and in the language attitudes literature.

Listeners in the survey heard one recording each from all eight speakers, in one of two possible orders, one randomly generated and the other its reverse. At the beginning of the survey, participants filled out a brief demographic question- 


\section{Kathryn Campbell-Kibler}

naire asking for their age, the school they attended, the countries or states they had lived in, and their racial or ethnic identity. After this preliminary page, listeners responded to eight survey pages, one for each recording. The survey form had six sections. The first was a set of adjective scales ranging from 1 to 6 , shown in (4). Note that the last item was labeled either masculine or feminine according to the gender of the speaker. Although these qualities are by no means mirror images of each other, time constraints necessitated that I limit myself to only one per speaker.

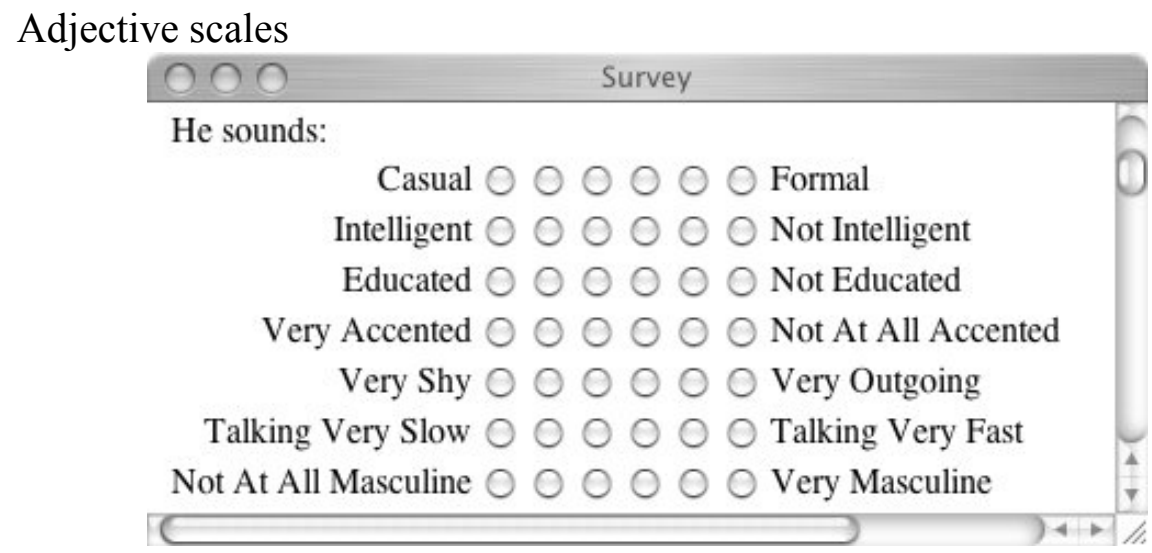

In the second section, listeners were asked to indicate how old the speaker sounded, given the choice of five checkboxes labeled with different age ranges: teenager, college-age, under 30 , in his/her 30 s, and over 40 . Listeners were required to select at least one age description, but could select as many as they chose in any combination. The next section contained an adjective checklist listing a range of personal qualities, such as lazy and religious, and social categories or professions, such as redneck and artist. After these personal descriptions came questions relating to the context. There was another six-point scale asking how well the speaker knew the addressee, ranging from best friend to stranger. Another set of checkboxes asked about speech activities, such as joking and chatting, and stances, such as bored and polite.

Lastly, the listeners were asked to guess the background of the speaker. Eight options for region were provided: the South, New England, the Midwest, the West Coast, the East Coast, the Southwest, the North, and anywhere. There were also options referring to community type: whether the speaker was from the city, the country, or the suburbs. The last set of boxes asked about class background: whether the speaker was from a working class background, a middle class background, or a wealthy background. In each of the checkbox sections except for age, one of the boxes labeled 'other' provided listeners with the opportunity to add their own descriptors. At the end of the page, an open-ended question invited them to add any remaining thoughts they had about the speaker. After listening to 


\section{Methods for the Study of Linguistic Variation}

all eight speakers, listeners were presented with a page giving options involving incentives and giving them an opportunity to send comments or feedback.

The survey itself was administered over the World Wide Web, allowing for a wider geographic distribution on a smaller budget. The length of the survey was adjusted to allow listeners to complete the entire process in approximately 15 minutes. This included reading over the consent form, filling out the demographic questionnaire and completing eight individual web forms, one for each speaker. A total of 124 participants completed the study. An additional 36 began it but failed to finish, and their data were removed from the analyses.

\section{Structure of the Data}

These procedures created a data set of such richness that, with the number of participants I had, I was able to only scratch the surface of the interactions and connections present. Different statistical techniques were needed for examining the three different categories of variables involved: independent variables, checkbox variables and rating variables. The independent variables gave information about which recording was being evaluated (the speaker, the recording itself, and the (ING) variant) and about the listener (school, gender, regional background, and race). The checkbox variables were binary (or nominal) variables indicating which yes/no attributes the listener had selected (e.g. articulate, artist). The ratings variables were numerical (or interval level) variables, ranging in number from 1 to 6 (e.g. not at all educated/very educated), indicating the listeners' selections on these scales.

I used the Chi Square test to investigate the influence of the independent variables on the checkbox variables, as well as levels of co-occurrence between the checkbox variables. To analyze the ratings variables, I used analysis of variance (ANOVA). These analyses examined both the influence of the independent variables on ratings and the relationship between checkbox variables and rating variables. I accomplished this latter task by temporarily treating a checkbox variable as an "independent" variable and using it as a term in an ANOVA run. Because of this, I used a hierarchical approach to the analysis of variance in which the order of the independent variables (or those being treated as independent for the purposes of the ANOVA) are considered in an order specified by the analyst. Thus, if the variable indicating which recording is being evaluated is listed first, it will be considered first; then its effects will be subtracted out of the analysis for the next term listed.

The bulk of the variables used in my analysis of variance fell into a clear logical hierarchy. The speaker in the recording or the choice of recording itself clearly accounted for the lion's share of variance in nearly all the responses, since listeners were most influenced in their judgments by which speaker they heard talking and what the person said. These two variables were the first terms. The next term in every round was (ING), then the listener characteristics: gender, race, school and regional background. These variables are much more difficult to rank in a theoretically justifiable way. However, the size of the data set made it difficult to 


\section{Kathryn Campbell-Kibler}

explore interactions between more than one of these in any case, so only one was included in each round of ANOVA.

The last possible category of terms is the checkbox variables discussed above. Although these variables are, strictly speaking, dependent variables, it is useful to treat them statistically as independent variables in some cases to explore the relationship between them and the ratings. In such cases, it is important to avoid assuming that one of these variables causes changes in the other. Because both are dependent variables, it is impossible to determine what causal relationship, if any, exists between them. Also, it is necessary for the checkbox terms to be considered last in the ANOVA run so as to avoid attributing a direct connection in a case where the two descriptions are merely correlated by being used to refer to the same speaker.

\section{New Insights}

The results of this study have been reported elsewhere, and the goal here is to present merely the methods. I will, however, touch on the general form of results in order to illustrate the ways that this technique has allowed us to answer previously unasked questions. In particular, it allows us to look not only at the effect of (ING) on single responses, but on interactions between multiple perceptions.

These interactions reveal a phenomenon I call "peak points", which occur when (ING) and one or more perceptions combine to create peaks with respect to another perception. An example of this may be seen in (5), which shows an interaction between (ING), intelligence, and the age category "in his/her 30s": speakers were rated significantly more intelligent when they used -ing and also were perceived as being in their 30s. When one element was missing, the other had no effect on intelligence. Listeners described age by selecting five checkboxes, and although overall age selections showed some normal curves for most qualities, the interaction was special to this one category. I believe this relates to the perception of the thirties by college students in terms of life and career path, particularly once the category of working class is introduced. (6) shows that the three primary choices - (ING), "working class", and "in his/her 30s"-combine to create a peak and a trough that are greater than the sum of each effect individually.

$$
\begin{aligned}
& \text { Intelligence ratings by (ING) and "in his/her 30s" } \\
& \begin{array}{ccc} 
& \text {-in } & \text {-ing } \\
\text { not in } 30 \mathrm{~s} & 3.75 & 3.73 \\
\text { in 30s } & 3.88 & 4.24 \\
(\mathrm{p}=0.006)
\end{array}
\end{aligned}
$$




\begin{tabular}{cl|r|r} 
& & -in & -ing \\
\hline \multirow{2}{*}{ not in his/her 30s s } & not working class & 3.86 & 3.73 \\
& working class & 3.22 & 3.74 \\
\hline \multirow{2}{*}{ in his/her 30s } & not working class & 4.00 & 4.45 \\
& working class & 3.50 & 3.65
\end{tabular}

This pattern and others like it suggest that we need to think about the relationship between language and social perceptions in an entirely new way and to start asking questions which can only be answered by using methods like these, which combine attention to social detail with experimental techniques.

\section{References}

Apple, William, Lynn A. Streeter, and Robert M. Krauss. 1979. Effects of Pitch and Speech Rate on Personal Attributions. Journal of Personality and Social Psychology 37:715-727.

Brown, B. L., W. J. Strong, and A. C. Rencher. 1973. Perception of Acoustical Parameters. Journal of the Acoustical Society of America 54:29-35.

Campbell-Kibler, Kathryn. Forthcoming. Variation and the Listener: The Contextual Meanings of (ING). In Penn Working Papers in Linguistics: Selected Papers from NWAV 34, Volume 12.2.

Dixon, John A., Berenice Mahoney, and Roger Cocks. 2002. Accents of Guilt? Effects of Regional Accent, Race, and Crime Type on Attributions of Guilt. Journal of Language and Social Psychology 21:162-168.

Fridland, Valerie, Kathryn Bartlett, and Roger Kreuz. 2004. Do You Hear What I Hear? Language Variation and Change 16:1-16.

Genesee, Fred, and Naomi Holobow. 1989. Change and Stability in Intergroup Perceptions. Journal of Language and Social Psychology 8:17-38.

Giles, Howard, Nikolas Coupland, Karen Henwood, Jim Harriman, and Justine Coupland. 1990. The Social Meaning of RP: An Intergenerational Perspective. In S. Ramgaran, ed., Studies in the Pronunciation of English: A Commemorative Volume in Honor of A. C. Gimson, 191-211. New York: Routledge.

Giles, Howard, Karen Henwood, Nikolas Coupland, Jim Harriman, and Justine Coupland. 1992. Language Attitudes and Cognitive Mediation. Human Communication Research 18:500-527.

Giles, Howard, Philip Smith, Caroline Browne, Sarah Whiteman, and Jennifer Williams. 1980. Women's Speech: The Voice of Feminism. In S. McConnell-Ginet, R. Borker, and N. Furman, eds., Women and Language in Literature and Society, 150-156. New York: Praeger. 


\section{Kathryn Campbell-Kibler}

Giles, Howard, and Robert N. St. Clair, eds. 1979. Language and Social Psychology. Oxford: Basil Blackwell.

Graff, David, William Labov, and Wendell A. Harris. 1986. Testing Listeners' Reactions to Phonological Markers of Ethnic Identity: A New Method For Sociolinguistic Research. In D. Sankoff, ed., Diversity and Diachrony, 45-58. Philadelphia: John Benjamins.

Hirose, Keikichi, and Hiromichi Kawanami. 2002. Temporal Rate Change of Dialogue Speech in Prosodic Units as Compared to Read Speech. Speech Communication 36:97-111.

Labov, William, Sharon Ash, Maciej Baranowski, Maya Ravindranath, and Tracy Weldon. 2005. Listeners' Sensitivity to the Frequency of Sociolinguistic Variables. Paper presented at New Ways of Analyzing Variation 34.

Lambert, Wallace E., R. C. Hodgson, R. C. Gardner, and S. Fillenbaum. 1960. Evaluational Reactions to Spoken Languages. Journal of Abnormal and Social Psychology 60.

Levin, Harry, Howard Giles, and Peter Garrett. 1994. The Effects of Lexical Formality and Accent on Trait Attributions. Language and Communication 14:211-236.

Plichta, Bartek, and Dennis R. Preston. 2005. The /ay/s Have It: The Perception of /ay/ as a North-South Stereotype in US English. Acta Linguistica Hafniensia, 243-285.

Scherer, Klaus R. 1979. Voice and Speech Correlates of Perceived Social Influence in Simulated Juries. In H. Giles and R. N. St. Clair, eds., Language and Social Psychology, 88-120.

Shuy, Roger W., and Ralph W. Fasold, eds. 1973. Language Attitudes: Current Trends and Prospects. Washington, DC: Georgetown University Press.

Smith, M. K., and G. H. Bailey. 1980. Attitude and Activity: Contextual Constraints on Subjective Judgments. In H. Giles, W. Robinson, and P. Smith, eds., Language: Social Psychological Perspectives, 209-226. Oxford: Pergamon Press.

Williams, Frederick, Nancy Hewett, Robert Hopper, Leslie M. Miller, Rita C. Naremore, and Jack L. Whitehead. 1976. Explorations of the Linguistic Attitudes of Teachers. Rowley, MA: Newbury House.

Wölck, Wolfgang. 1973. Attitudes toward Spanish and Quechua in Bilingual Peru. In R. W. Shuy and R. W. Fasold, eds., Language Attitudes: Current Trends and Prospects, 129-147.

Kathryn Campbell-Kibler

Department of Linguistics

Stanford University

Stanford, CA 94305-2150

kcat@csli.stanford.edu 